\title{
DEFORMATIONS WITH CONSTANT LÊ NUMBERS AND MULTIPLICITY OF NONISOLATED HYPERSURFACE SINGULARITIES
}

\author{
CHRISTOPHE EYRAL AND MARIA APARECIDA \\ SOARES RUAS
}

\begin{abstract}
We show that the possible jump of the order in an 1-parameter deformation family of (possibly nonisolated) hypersurface singularities, with constant Lê numbers, is controlled by the powers of the deformation parameter. In particular, this applies to families of aligned singularities with constant topological type - a class for which the Lê numbers are "almost" constant. In the special case of families with isolated singularities - a case for which the constancy of the Lê numbers is equivalent to the constancy of the Milnor number - the result was proved by Greuel, Plénat, and Trotman.

As an application, we prove equimultiplicity for new families of nonisolated hypersurface singularities with constant topological type, answering partially the Zariski multiplicity conjecture.
\end{abstract}

\section{$\S 1$. Introduction}

Let $B$ and $D$ be open balls around the origins in $\mathbb{C}^{n}(n \geq 2)$ and $\mathbb{C}$, respectively, let $\mathbf{z}:=\left(z_{1}, \ldots, z_{n}\right)$ be linear coordinates for $\mathbb{C}^{n}$, and let

$$
f:(B \times D,\{\mathbf{0}\} \times D) \rightarrow(\mathbb{C}, 0), \quad(\mathbf{z}, t) \mapsto f_{t}(\mathbf{z}):=f(\mathbf{z}, t)
$$

be a holomorphic function. We suppose that, for all $t \in D$, the function $f_{t}$ is reduced. We denote by $\operatorname{mult}\left(f_{t}\right)$ the multiplicity of $V\left(f_{t}\right):=f_{t}^{-1}(0)$ at $\mathbf{0} \in \mathbb{C}^{n}$, that is, the number of points of intersection, near $\mathbf{0}$, of $V\left(f_{t}\right)$ with a generic line in $\mathbb{C}^{n}$ passing arbitrarily close to, but not through, the origin. As $f_{t}$ is reduced, $\operatorname{mult}\left(f_{t}\right)$ is also the order of $f_{t}$ at $\mathbf{0}$ (denoted by $\operatorname{ord}\left(f_{t}\right)$ ) - that is, the lowest degree in the power series expansion of $f_{t}$ at $\mathbf{0}$.

One says that the family $\left\{f_{t}\right\}$ is topologically $V$-constant if, for all sufficiently small $t$, there exist open neighborhoods $U_{0}, U_{t} \subseteq B$ around the origin

Received March 9, 2013. Revised September 2, 2013. Accepted November 4, 2013. First published online December 11, 2014.

2010 Mathematics Subject Classification. Primary 32S15; Secondary 32S25, 32S05. 
together with a homeomorphism $\varphi_{t}:\left(U_{t}, \mathbf{0}\right) \rightarrow\left(U_{0}, \mathbf{0}\right)$ such that $\varphi_{t}\left(V\left(f_{t}\right) \cap\right.$ $\left.U_{t}\right)=V\left(f_{0}\right) \cap U_{0}$. One says that $\left\{f_{t}\right\}$ is equimultiple if, for all sufficiently small $t$, the multiplicity $\operatorname{mult}\left(f_{t}\right)$ is independent of $t$ (i.e., if $\operatorname{mult}\left(f_{t}\right)=$ $\left.\operatorname{mult}\left(f_{0}\right)\right)$.

Conjecture 1.1 (Zariski's multiplicity conjecture [20]). If the family $\left\{f_{t}\right\}$ is topologically $V$-constant, then it is equimultiple.

In fact, to be precise, Zariski did not so much conjecture as ask whether the topological $V$-constancy implies equimultiplicity. Nevertheless, it is usual (and convenient) to call Zariski's original question the Zariski multiplicity conjecture. By the Lê-Ramanujam theorem [11], in the special case of families with isolated singularities, and provided that $n \neq 3$, the Zariski multiplicity conjecture is equivalent to the following conjecture by Teissier [18].

Conjecture 1.2 (Teissier). Suppose that, for all $t$ sufficiently small, $f_{t}$ has an isolated singularity at the origin. If the family $\left\{f_{t}\right\}$ is $\mu$-constant (i.e., if, for all sufficiently small $t$, the Milnor number of $f_{t}$ at $\mathbf{0}, \mu_{f_{t}}(\mathbf{0})$, is independent of $t$ ), then it is equimultiple.

Although partial positive answers have been given regularly, Zariski's conjecture (as well as Teissier's conjecture) is, in general, still unsettled. (For a list of the main known results, we refer the reader to [4].)

Write

$$
f_{t}(\mathbf{z})=f_{0}(\mathbf{z})+\sum_{j \geq 1} t^{j} g_{j}(\mathbf{z}),
$$

where $g_{j}: B \rightarrow \mathbb{C}$ is a holomorphic function. Clearly, the family $\left\{f_{t}\right\}$ is not equimultiple if and only if there exists $j_{1}$ with $g_{j_{1}} \neq 0$ and

$$
\inf _{j \geq 1} \operatorname{ord}\left(g_{j}\right)<\operatorname{ord}\left(f_{0}\right)=\operatorname{mult}\left(f_{0}\right)
$$

Hereafter, we will always assume that there is an index $j_{1}$ such that $g_{j_{1}} \neq 0$. We will also suppose that $f_{0} \neq 0$. In [6] and [16], Greuel, Plénat, and Trotman showed that the possible jump in the order in a $\mu$-constant family of isolated hypersurface singularities is controlled by the powers of $t$. Precisely, they proved that $\operatorname{ord}\left(g_{j}\right) \geq \operatorname{ord}\left(f_{0}\right)-j+1$ for all $j \geq 1$. (If $g_{j}=0$, then, by convention, we set $\operatorname{ord}\left(g_{j}\right)=\infty$ so that the inequality above remains valid.) In the present paper, we will show that this estimation of the order's jump 
also holds for families of nonisolated hypersurface singularities, provided the $\mu$-constancy is replaced by the constancy of the Lê numbers with respect to the coordinates $\mathbf{z}$ (which we will call the $\lambda_{\mathbf{z}}$-constancy). Note that, in the special case of a family with isolated singularities, $\lambda_{\mathbf{z}}$-constant means exactly $\mu$-constant. Concerning the proofs, for families of isolated singularities, the argument is based on the Lê-Saito-Teissier valuation test for $\mu$-constancy (see [12], [18]). For nonisolated singularities, there are two different ways to get the result. We may either invoke a generalization of the Lê-SaitoTeissier theorem due to Massey [13] or we can apply a generalization of the Iomdine-Lê formula also due to Massey [13].

Using the classical Lê-Saito-Teissier theorem, Plénat and Trotman in [16] and Greuel and Pfister in [7] were also able to obtain equimultiplicity for certain families of isolated singularities. For example, it is proved in [16] that $\mu$-constant families of isolated singularities of the form $f_{t}(\mathbf{z})=$ $f_{0}(\mathbf{z})+t g_{1}(\mathbf{z})+t^{2} g_{2}(\mathbf{z})$ are equimultiple if the singular locus of the tangent cone to $V\left(f_{0}\right)$ is not contained in the tangent cone to $V\left(g_{2}\right)$. In [7], it is shown, among other things, that any $\mu$-constant family of isolated singularities of the form $f_{t}\left(z_{1}, \ldots, z_{n}\right)=h_{t}\left(z_{1}, \ldots, z_{n-1}\right)+z_{n}^{2} k_{t}\left(z_{1}, \ldots, z_{n}\right)$ is equimultiple if $h_{0}$ is semiquasihomogeneous (or if $n=3$ ) and $\operatorname{ord}\left(h_{t}\right) \leq \operatorname{ord}\left(z_{n}^{2} k_{t}\right)$. Using the general Massey-Lê-Saito-Teissier theorem and Massey-IomdineLê formula, we show in the following pages that statements similar to those of [16] and [7] also hold for nonisolated singularities, provided that the $\mu$ constancy is replaced by the $\lambda_{\mathbf{z}}$-constancy.

\section{$\S 2$. $\lambda_{\mathbf{z}}$-constant deformations and Thom's $a_{f}$ condition}

Let $\Sigma f_{t}$ be the critical locus of $f_{t}$. Throughout this paper, we assume that $\operatorname{dim}_{\mathbf{0}} \Sigma f_{t}$ is greater than or equal to 0 (i.e., $f_{t}$ has a critical point at $\mathbf{0}$ ) and is constant as $t$ varies. We write $d:=\operatorname{dim}_{0} \Sigma f_{t}$.

We say that the family $\left\{f_{t}\right\}$ is $\lambda_{\mathbf{z}}$-constant (or $\lambda$-constant with respect to the coordinates $\left.\mathbf{z}=\left(z_{1}, \ldots, z_{n}\right)\right)$ if, for all $0 \leq i \leq d$ and for all sufficiently small $t$, the $i$ th Lê number of $f_{t}$ at $\mathbf{0}$ with respect to $\mathbf{z}, \lambda_{f_{t}, \mathbf{z}}^{i}(\mathbf{0})$, is defined and independent of $t$. (For the definition of Lê's numbers, see [13], [14]. For the convenience of the reader, we also recall the definition in Appendix A.) Note that, when $d=0$ (i.e., for isolated singularities), the Lê number $\lambda_{f_{t}, \mathbf{z}}^{0}(\mathbf{0})$ is nothing but the Milnor number of $f_{t}$ at $\mathbf{0}$ (see [13, Example 2.1]). Therefore, in this case, the family $\left\{f_{t}\right\}$ is $\lambda_{\mathbf{z}}$-constant if and 
only if it is $\mu$-constant. It is well known that topologically $V$-constant families of isolated hypersurface singularities are $\mu$-constant (see [10], [18]). In general, for nonisolated singularities, it is not true that the topological $V$ -

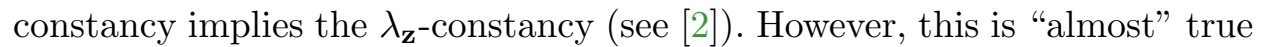
for a special class of singularities called aligned singularities. ${ }^{\dagger}$ More precisely, if $\left\{f_{t}\right\}$ is a topologically $V$-constant family of aligned singularities and if $\left\{t_{k}\right\}$ is an infinite sequence of points in $\mathbb{C}$ approaching the origin, then, as explained in the proof of [13, Theorem 7.9], we can use the Baire category theorem to conclude that there exists an aligning set of coordinates (see [13, Definition 7.1]) for $f_{0}$ and for $f_{t_{k}}$ for all $k$. (The existence of a set of coordinates which is aligning for $f_{t}$ for all small $t$ is not always clear.) Then, if we still denote by $\mathbf{z}$ the new coordinates system, Corollary 7.8 of [13] implies that $\lambda_{f_{t_{k}}, \mathbf{z}}^{i}(\mathbf{0})=\lambda_{f_{0}, \mathbf{z}}^{i}(\mathbf{0})$. In other words, for aligned singularities, the topological $V$-constancy implies the constancy of the Lê numbers (with respect to an appropriate coordinates system) at least along a sequence $\left\{f_{0}, f_{t_{1}}, \ldots, f_{t_{k}}, \ldots\right\}$ within the family $\left\{f_{t}\right\}$, where $t_{k} \rightarrow 0$.

In [13], Massey proved the following generalization of the Lê-Saito-Teissier theorem.

Theorem 2.1 ([13, Theorem 6.5]). If the family $\left\{f_{t}\right\}$ is $\lambda_{\mathbf{z}}$-constant, then $\{\mathbf{0}\} \times D$ satisfies Thom's $a_{f}$ condition at the origin with respect to the ambient stratum, that is, if $\left\{\mathbf{p}_{k}\right\}$ is a sequence of points in $(B \times D) \backslash \Sigma f$ such that $\mathbf{p}_{k} \rightarrow(\mathbf{0}, 0)$ and $T_{\mathbf{p}_{k}} V\left(f-f\left(\mathbf{p}_{k}\right)\right) \rightarrow T$, then $\{\mathbf{0}\} \times \mathbb{C}=T_{(\mathbf{0}, 0)}(\{\mathbf{0}\} \times$ $D) \subseteq T$.

As usual, $T_{\mathbf{p}_{k}} V\left(f-f\left(\mathbf{p}_{k}\right)\right)$ denotes the tangent space at $\mathbf{p}_{k}$ to the level hypersurface in $\mathbb{C}^{n+1}$ defined by $f(\mathbf{z}, t)=f\left(\mathbf{p}_{k}\right)$, and $T_{(\mathbf{0}, 0)}(\{\mathbf{0}\} \times D)$ denotes the tangent space at $(\mathbf{0}, 0)$ to $\{\mathbf{0}\} \times D$. The notation $\Sigma f$ stands for the critical locus of $f$. In the special case where $\left\{f_{t}\right\}$ is a family of isolated singularities, since $\lambda_{\mathbf{z}}$-constant simply means $\mu$-constant, Theorem 2.1 coincides with the classical Lê-Saito-Teissier theorem.

LEMma 2.2. If $\{\mathbf{0}\} \times D$ satisfies Thom's $a_{f}$ condition at the origin with respect to the ambient stratum, then, for any holomorphic curve

$$
\gamma:(\mathbb{C}, 0) \rightarrow(B \times D,(\mathbf{0}, 0)), \quad s \mapsto \gamma(s),
$$

\footnotetext{
$\dagger$ These singularities were introduced by Massey in [13, Definition 7.1]. They include, for instance, isolated singularities and line singularities. We recall the definition in Appendix B.
} 
not contained ${ }^{\dagger}$ in $\Sigma f$, we have

$$
\operatorname{ord}\left(\frac{\partial f}{\partial t} \circ \gamma\right)>\inf _{1 \leq i \leq n} \operatorname{ord}\left(\frac{\partial f}{\partial z_{i}} \circ \gamma\right)
$$

where ord $(\cdot)$ is the order in $s$ at 0 .

Definition 2.3. Hereafter, we will say that $f$ satisfies the Thom inequalities if, for any holomorphic curve $\gamma$ as in Lemma 2.2 (i.e., $\gamma(0)=(\mathbf{0}, 0)$ and $\gamma(\mathbb{C}) \nsubseteq \Sigma f)$, the inequality (2.1) holds.

Proof of Lemma 2.2. We argue by contradiction. Suppose that there is a holomorphic curve $\gamma:(\mathbb{C}, 0) \rightarrow(B \times D,(\mathbf{0}, 0))$ not contained in $\Sigma f$ and such that (2.1) does not hold. Then, the limit of the projective class of

$$
\operatorname{grad} f(\gamma(s)):=\left(\frac{\partial f}{\partial z_{1}}(\gamma(s)), \ldots, \frac{\partial f}{\partial z_{n}}(\gamma(s)), \frac{\partial f}{\partial t}(\gamma(s))\right),
$$

as $s$ tends to 0 , has the form $\left[v_{1}: \cdots: v_{n}: w\right]$ with $w \neq 0$, and hence $\{\mathbf{0}\} \times D$ does not satisfy Thom's $a_{f}$ condition at the origin along the curve $\gamma$.

Combining Theorem 2.1 and Lemma 2.2 gives the following corollary.

Corollary 2.4. If the family $\left\{f_{t}\right\}$ is $\lambda_{\mathbf{z}}$-constant, then $f$ satisfies the Thom inequalities (see Definition 2.3).

\section{$\S 3$. Possible jump of the order in a $\lambda_{z}$-constant family}

In [6, Lemma 1.3] and [16, Proposition 2.2], Greuel, Plénat, and Trotman showed that the possible jump in the order in a $\mu$-constant family of isolated hypersurface singularities $\left\{f_{t}\right\}$ is controlled by the powers of $t$. The precise statement is the following.

THEOREM 3.1. Suppose that, for all t sufficiently small, $f_{t}$ has an isolated singularity at the origin. In this case, if the family $\left\{f_{t}\right\}$ is $\mu$-constant, then, for all $j \geq 1$,

$$
\operatorname{ord}\left(g_{j}\right) \geq \operatorname{ord}\left(f_{0}\right)-j+1 \text {. }
$$

In the next theorem, we show that this estimation of the order's jump also holds for any family of nonisolated hypersurface singularities, provided that the $\mu$-constancy is replaced by the $\lambda_{\mathbf{z}}$-constancy.

\footnotetext{
† Throughout, for any subsets $A, B \subseteq \mathbb{C}^{n}$ containing $\mathbf{0}$, by " $A \nsubseteq B$ " we mean that $A \nsubseteq B$ in an arbitrarily small neighborhood of $\mathbf{0}$. Similarly, for any function $h$, by " $h \neq 0$ " we mean $h \neq 0$ near the origin.
} 
Theorem 3.2. If the family $\left\{f_{t}\right\}$ is $\lambda_{\mathbf{z}}$-constant, then, for all $j \geq 1$,

$$
\operatorname{ord}\left(g_{j}\right) \geq \operatorname{ord}\left(f_{0}\right)-j+1 \text {. }
$$

We give two proofs of Theorem 3.2. The first one is on the same pattern as the proof of [6, Lemma 1.3] and [16, Proposition 2.2] with the classical Lê-Saito-Teissier theorem replaced by the general version of Massey. With this proof, Theorem 3.1 appears as a corollary of Theorem 3.2. In fact, this proof shows that if $f$ satisfies the Thom inequalities (see Definition 2.3), then $\operatorname{ord}\left(g_{j}\right) \geq \operatorname{ord}\left(f_{0}\right)-j+1$ for all $j \geq 1$. In the second proof, we deduce Theorem 3.2 from Theorem 3.1 thanks to a general version of the IomdineLê formula also due to Massey (see [13, Theorem 4.5, Corollary 4.6]). With this proof, the tools required to prove Theorem 3.2 involve both the LêSaito-Teissier theorem (through Theorem 3.1) and the Massey-Iomdine-Lê formula. Unlike the first proof, with this second approach one really needs to assume that the family $\left\{f_{t}\right\}$ is $\lambda_{\mathbf{z}}$-constant in order to apply the MasseyIomdine-Lê formula (it is not enough to simply assume that $f$ satisfies the Thom inequalities shown in (2.1)).

Notation 3.3. Hereafter, if $h$ is any holomorphic function near $\mathbf{0}$, then we will denote by in $(h)$ its initial polynomial at $\mathbf{0}$.

First proof of Theorem 3.2. Consider the partial derivatives

$$
\frac{\partial f}{\partial t}=\sum_{j \geq 1} j t^{j-1} g_{j} \quad \text { and } \quad \frac{\partial f}{\partial z_{i}}=\frac{\partial f_{0}}{\partial z_{i}}+\sum_{j \geq 1} t^{j} \frac{\partial g_{j}}{\partial z_{i}} .
$$

Choose an index $i_{0}$ such that $\partial f / \partial z_{i_{0}} \neq 0$, and pick a point $\left(\mathbf{z}_{0}, t_{0}\right) \in(B \backslash$ $\{\mathbf{0}\}) \times(D \backslash\{0\})$ such that, for all $s \neq 0$ sufficiently small,

$$
\operatorname{in}\left(\frac{\partial f}{\partial t}\right)\left(s \mathbf{z}_{0}, s t_{0}\right) \neq 0 \quad \text { and } \quad \operatorname{in}\left(\frac{\partial f}{\partial z_{i_{0}}}\right)\left(s \mathbf{z}_{0}, s t_{0}\right) \neq 0 \text {. }
$$

Then, if $\gamma:(\mathbb{C}, 0) \rightarrow(B \times D,(\mathbf{0}, 0))$ is the curve defined by $s \mapsto \gamma(s)=$ $\left(\gamma_{1}(s), \gamma_{2}(s)\right)=\left(s \mathbf{z}_{0}, s t_{0}\right)$, we have

$$
\operatorname{ord}\left(\frac{\partial f}{\partial t} \circ \gamma\right)=\operatorname{ord}\left(\frac{\partial f}{\partial t}\right)=\inf _{j \geq 1}\left(j-1+\operatorname{ord}\left(g_{j}\right)\right),
$$

while

$$
\begin{aligned}
\inf _{1 \leq i \leq n} \operatorname{ord}\left(\frac{\partial f}{\partial z_{i}} \circ \gamma\right) & \geq \inf _{\substack{1 \leq i \leq n \\
j \geq 1}}\left\{\operatorname{ord}\left(\frac{\partial f_{0}}{\partial z_{i}} \circ \gamma_{1}\right), \operatorname{ord}\left(\gamma_{2}^{j} \cdot \frac{\partial g_{j}}{\partial z_{i}} \circ \gamma_{1}\right)\right\} \\
& \geq \inf \left\{\operatorname{ord}\left(f_{0}\right)-1, \inf _{j \geq 1}\left(j+\operatorname{ord}\left(g_{j}\right)-1\right)\right\} .
\end{aligned}
$$


As $\operatorname{in}\left(\partial f / \partial z_{i_{0}}\right) \circ \gamma \neq 0$, the set $\gamma(\mathbb{C})$ is not contained in $\Sigma f$, and Corollary 2.4 implies that $\operatorname{ord}\left(f_{0}\right)-1<j+\operatorname{ord}\left(g_{j}\right)-1$ for all $j \geq 1$.

Second proof of Theorem 3.2. Here, we proceed as in the proof of Theorem 7.9 in [13]. As $\left\{f_{t}\right\}$ is $\lambda_{\mathbf{z}}$-constant, [13, Corollary 4.6] shows that, for all integers $0 \ll N_{1} \ll N_{2} \ll \cdots \ll N_{d}$ (where $d=\operatorname{dim}_{0} \Sigma f_{t}$ ), the functions $f_{0}+z_{1}^{N_{1}}+\cdots+z_{d}^{N_{d}}$ and $f_{t}+z_{1}^{N_{1}}+\cdots+z_{d}^{N_{d}}$ have an isolated singularity at $\mathbf{0}$ and the same Milnor number for all $t$ sufficiently small. That is, $\left\{f_{t}+z_{1}^{N_{1}}+\cdots+z_{d}^{N_{d}}\right\}$ is a $\mu$-constant family of isolated singularities. Therefore, by Theorem 3.1, we have

$$
\operatorname{ord}\left(g_{j}\right) \geq \operatorname{ord}\left(f_{0}+z_{1}^{N_{1}}+\cdots+z_{d}^{N_{d}}\right)-j+1 .
$$

As $N_{1}, \ldots, N_{d}$ are arbitrarily large, we deduce that $\operatorname{ord}\left(g_{j}\right) \geq \operatorname{ord}\left(f_{0}\right)-j+1$.

Theorem 3.2 has the following immediate corollary.

Corollary 3.4. Suppose that $f_{t}$ is of the form $f_{t}(\mathbf{z})=f_{0}(\mathbf{z})+t g_{1}(\mathbf{z})$. In this case, if the family $\left\{f_{t}\right\}$ is $\lambda_{\mathbf{z}}$-constant, then it is equimultiple.

More generally, we have the next result.

Corollary 3.5. Suppose that $f_{t}$ can be written in the form $f_{t}(\mathbf{z})=$ $f_{0}(\mathbf{z})+\xi(t) g(\mathbf{z})$, where $\xi:(D, 0) \rightarrow(\mathbb{C}, 0)$ is a nonconstant holomorphic function and where $g:(B, \mathbf{0}) \rightarrow(\mathbb{C}, 0)$ is any holomorphic function. In this case, if the family $\left\{f_{t}\right\}$ is $\lambda_{\mathbf{z}}$-constant, then it is equimultiple. (In particular, any $\lambda_{\mathbf{z}}$-constant family of the form $f_{t}(\mathbf{z})=f_{0}(\mathbf{z})+t^{j} g_{j}(\mathbf{z})$ is equimultiple.)

In the special case of families with isolated singularities - a case for which the $\lambda_{\mathbf{z}}$-constancy is the same as the $\mu$-constancy-Corollaries 3.4 and 3.5 were first proved by Greuel in [6, Lemma 1.2] and by Trotman [19, Corollary 2].

Proof of Corollary 3.5. Consider the deformation family $h_{t}(\mathbf{z}):=f_{0}(\mathbf{z})+$ $\operatorname{tg}(\mathbf{z})$. As $\xi$ is a nonconstant holomorphic function, it is open, and there-

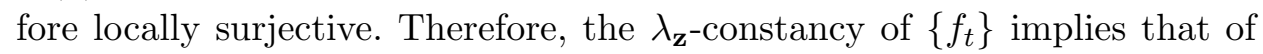
$\left\{h_{t}\right\}$. Hence, by Corollary 3.4, $\left\{h_{t}\right\}$ is equimultiple. Clearly, this implies the equimultiplicity of $\left\{f_{t}\right\}$.

Concerning topologically $V$-constant families of aligned singularities, Theorems 3.1 and 3.2 also have the following corollary. 
Corollary 3.6. Suppose that, for all sufficiently small t, the function $f_{t}$ has a d-dimensional aligned singularity at $\mathbf{0}$. In this case, if the family $\left\{f_{t}\right\}$ is topologically $V$-constant, then, for all $j \geq 1$,

$$
\operatorname{ord}\left(g_{j}\right) \geq \operatorname{ord}\left(f_{0}\right)-j+1 \text {. }
$$

Proof. If there exists a set of coordinates which is aligning for $f_{t}$ at $\mathbf{0}$, for all sufficiently small $t$, then, by [13, Corollary 7.8], the family $\left\{f_{t}\right\}$ is $\lambda$-constant with respect to this coordinates system, and the result follows immediately from Theorem 3.2. Otherwise, we proceed as in the proof of Theorem 7.9 in [13]. We pick an infinite sequence of points $\left\{t_{k}\right\}$ in $\mathbb{C}$ approaching the origin, and we apply the Baire category theorem to conclude that there exists a set of coordinates (that we will still denote by z) which is aligning for $f_{0}$ and for $f_{t_{k}}$ for all $k$. Then, by [13, Corollary 7.8], $\lambda_{f_{t_{k}}, \mathbf{z}}^{i}(\mathbf{0})=\lambda_{f_{0}, \mathbf{z}}^{i}(\mathbf{0})$ for all large $k$. Hence, by [13, Corollary 4.6], for all integers $0 \ll N_{1} \ll N_{2} \ll \cdots \ll N_{d}$, the functions $f_{0}+z_{1}^{N_{1}}+\cdots+z_{d}^{N_{d}}$ and $f_{t_{k}}+z_{1}^{N_{1}}+\cdots+z_{d}^{N_{d}}$ have an isolated singularity at $\mathbf{0}$ and the same Milnor number, provided that $k$ is large enough. By the upper semicontinuity of the Milnor number, this implies that, for all $t$ sufficiently small, $f_{t}+z_{1}^{N_{1}}+\cdots+z_{d}^{N_{d}}$ has an isolated singularity at $\mathbf{0}$ and the same Milnor number as $f_{0}+z_{1}^{N_{1}}+\cdots+z_{d}^{N_{d}}$. In other words, $\left\{f_{t}+z_{1}^{N_{1}}+\cdots+z_{d}^{N_{d}}\right\}$ is a $\mu$-constant family of isolated singularities. Therefore, by Theorem 3.1, we have

$$
\operatorname{ord}\left(g_{j}\right) \geq \operatorname{ord}\left(f_{0}+z_{1}^{N_{1}}+\cdots+z_{d}^{N_{d}}\right)-j+1 .
$$

As $N_{1}, \ldots, N_{d}$ are arbitrarily large, $\operatorname{ord}\left(g_{j}\right) \geq \operatorname{ord}\left(f_{0}\right)-j+1$.

In particular, we have the next equimultiplicity result.

Corollary 3.7. Suppose that $f_{t}$ can be written in the form $f_{t}(\mathbf{z})=$ $f_{0}(\mathbf{z})+\xi(t) g(\mathbf{z})$, where $\xi:(D, 0) \rightarrow(\mathbb{C}, 0)$ is a nonconstant holomorphic function and where $g:(B, \mathbf{0}) \rightarrow(\mathbb{C}, 0)$ is any holomorphic function. Suppose also that, for all sufficiently small $t$, the function $f_{t}$ has an aligned singularity at $\mathbf{0}$. Under these conditions, if the family $\left\{f_{t}\right\}$ is topologically $V$-constant, then it is equimultiple.

Proof. The result is immediate when $\xi(t)=t$ (see Corollary 3.6). When $\xi(t) \neq t$, then, as in the proof of Corollary 3.5, the result follows from the case where $\xi(t)=t$ and from the local surjectivity of $\xi$. 
§4. $\lambda_{\mathbf{z}}$-constant families of the form $f_{t}(\mathbf{z})=f_{0}(\mathbf{z})+t g_{1}(\mathbf{z})+t^{2} g_{2}(\mathbf{z})$

In this section, we focus on deformations of the form $f_{t}(\mathbf{z})=f_{0}(\mathbf{z})+$ $t g_{1}(\mathbf{z})+t^{2} g_{2}(\mathbf{z})$. Under this assumption, Plénat and Trotman proved the following equimultiplicity result.

Theorem 4.1 ([16, Proposition 3.2]). Suppose that $f_{t}(\mathbf{z})=f_{0}(\mathbf{z})+t g_{1}(\mathbf{z})+$ $t^{2} g_{2}(\mathbf{z})$, with $g_{2} \neq 0$. Suppose also that, for all sufficiently small $t$, the function $f_{t}$ has an isolated singularity at the origin. Under these conditions, if the family $\left\{f_{t}\right\}$ is $\mu$-constant and such that the singular locus $\Sigma \operatorname{in}\left(f_{0}\right)$ of the tangent cone to $V\left(f_{0}\right)$ is not contained in the tangent cone to $V\left(g_{2}\right)$, then $\left\{f_{t}\right\}$ is equimultiple.

We recall that the tangent cone $C\left(V\left(f_{0}\right)\right)$ to $V\left(f_{0}\right)$ is the zero set of the initial polynomial, in $\left(f_{0}\right)$, of $f_{0}$ at $\mathbf{0}$. Similarly, $C\left(V\left(g_{2}\right)\right)=\left(\operatorname{in}\left(g_{2}\right)\right)^{-1}(0)$.

In the next theorem, we show that this result also holds for families of nonisolated hypersurface singularities, provided that the constancy of the Milnor number is replaced by the constancy of the Lê numbers.

THEOREM 4.2. Suppose that $f_{t}(\mathbf{z})=f_{0}(\mathbf{z})+t g_{1}(\mathbf{z})+t^{2} g_{2}(\mathbf{z})$, with $g_{2} \neq 0$. In this case, if the family $\left\{f_{t}\right\}$ is $\lambda_{\mathbf{z}}$-constant and such that $\Sigma \operatorname{in}\left(f_{0}\right) \nsubseteq$ $C\left(V\left(g_{2}\right)\right)$, then it is equimultiple.

We also give two proofs of Theorem 4.2. The first one is on the same pattern as the proof of [16, Proposition 3.2], with the classical Lê-SaitoTeissier theorem replaced by Massey's general version. With this proof, Theorem 4.1 appears as a corollary of Theorem 4.2. In the second proof, we deduce Theorem 4.2 from Theorem 4.1 thanks to the Massey-IomdineLê formula. So, this proof requires the use of both the Lê-Saito-Teissier theorem and the Massey-Iomdine-Lê formula. Another advantage of the first proof compared with the second one is that, in Theorem 4.2, the $\lambda_{\mathbf{z}^{-}}$ constancy can actually be replaced by the condition " $f$ satisfies the Thom inequalities" (see Remarks 4.4 and 4.5).

To give the first proof of Theorem 4.2, we need the following lemma in which we do not assume that the family $\left\{f_{t}\right\}$ is $\lambda_{\mathbf{z}}$-constant.

LEMma 4.3. Suppose that $f_{t}(\mathbf{z})=f_{0}(\mathbf{z})+t g_{1}(\mathbf{z})+t^{2} g_{2}(\mathbf{z})$, with $g_{2} \neq 0$ and $\sin \left(f_{0}\right) \nsubseteq C\left(V\left(g_{2}\right)\right)$. Then,

$$
\sin \left(f_{0}\right) \times \mathbb{C} \nsubseteq \Xi f:=\left\{(\mathbf{z}, t) \in \mathbb{C}^{n} \times \mathbb{C} ; \frac{\partial f}{\partial z_{i}}(\mathbf{z}, t)=0,1 \leq i \leq n\right\}
$$


Proof. First of all, observe that $\operatorname{dim}_{0} \Sigma \operatorname{in}\left(f_{0}\right) \geq 1$. (If $\operatorname{dim}_{0} \Sigma \operatorname{in}\left(f_{0}\right)=0$, then $\Sigma \operatorname{in}\left(f_{0}\right)=\{\mathbf{0}\} \subseteq C\left(V\left(g_{2}\right)\right)$, which is a contradiction.) Now, suppose that $\operatorname{\Sigma in}\left(f_{0}\right) \times \mathbb{C} \subseteq \Xi f$, that is, for all $\mathbf{z} \in \Sigma i n\left(f_{0}\right), t \in \mathbb{C}$, and $1 \leq i \leq n$,

$$
\frac{\partial f}{\partial z_{i}}(\mathbf{z}, t)=\frac{\partial f_{t}}{\partial z_{i}}(\mathbf{z})=\frac{\partial f_{0}}{\partial z_{i}}(\mathbf{z})+\sum_{j=1}^{2} t^{j} \cdot \frac{\partial g_{j}}{\partial z_{i}}(\mathbf{z})=0 .
$$

In particular, this implies that, for all $\mathbf{z} \in \Sigma \operatorname{in}\left(f_{0}\right)$ and all $1 \leq i \leq n,\left(\partial g_{2} /\right.$ $\left.\partial z_{i}\right)(\mathbf{z})=0$, and consequently $g_{2}$ is constant on each stratum of $\Sigma \operatorname{in}\left(f_{0}\right)$. Clearly, $\mathbf{0} \in \Sigma \operatorname{in}\left(f_{0}\right)$ and $g_{2}$ vanishes on the stratum containing this point. Hence, by continuity, $g_{2}=0$ on each stratum, that is, $g_{2 \mid \Sigma \operatorname{in}\left(f_{0}\right)}=0$. This, in turn, implies that

$$
\operatorname{in}\left(g_{2}\right)_{\mid \Sigma i n\left(f_{0}\right)}=0
$$

which is a contradiction with the hypothesis $\Sigma \operatorname{in}\left(f_{0}\right) \nsubseteq C\left(V\left(g_{2}\right)\right)=$ $\left(\operatorname{in}\left(g_{2}\right)\right)^{-1}(0)$. To show that in $\left(g_{2}\right)_{\mid \sin \left(f_{0}\right)}=0$, write

$$
g_{2}(\mathbf{z})=\operatorname{in}\left(g_{2}\right)(\mathbf{z})+g_{2, d_{1}}(\mathbf{z})+g_{2, d_{2}}(\mathbf{z})+\cdots,
$$

where $g_{2, d_{k}}$ is a homogeneous polynomial of degree $d_{k}$ with deg $\operatorname{in}\left(g_{2}\right)<d_{1}<$ $d_{2}<\cdots$, and pick a point $\mathbf{z}_{0} \in \Sigma \operatorname{in}\left(f_{0}\right)$. For any $\zeta \in \mathbb{C}, \zeta \mathbf{z}_{0} \in \Sigma \operatorname{in}\left(f_{0}\right)$, and therefore

$$
0=g_{2}\left(\zeta \mathbf{z}_{0}\right)=\zeta^{\operatorname{deg} \operatorname{in}\left(g_{2}\right)} \operatorname{in}\left(g_{2}\right)\left(\mathbf{z}_{0}\right)+\zeta^{d_{1}} g_{2, d_{1}}\left(\mathbf{z}_{0}\right)+\zeta^{d_{2}} g_{2, d_{2}}\left(\mathbf{z}_{0}\right)+\cdots
$$

Now, if in $\left(g_{2}\right)\left(\mathbf{z}_{0}\right) \neq 0$, then, for $\zeta \neq 0$ sufficiently small,

$$
\left|\zeta^{\operatorname{deg} \operatorname{in}\left(g_{2}\right)} \operatorname{in}\left(g_{2}\right)\left(\mathbf{z}_{0}\right)\right| \gg\left|\zeta^{d_{1}} g_{2, d_{1}}\left(\mathbf{z}_{0}\right)+\zeta^{d_{2}} g_{2, d_{2}}\left(\mathbf{z}_{0}\right)+\cdots\right|,
$$

and consequently $g_{2}\left(\zeta \mathbf{z}_{0}\right) \neq 0$, which is a contradiction.

Now we are able to give the first proof of Theorem 4.2.

First proof of Theorem 4.2. By Theorem 3.2, $\operatorname{ord}\left(g_{1}\right) \geq \operatorname{ord}\left(f_{0}\right)$ and $\operatorname{ord}\left(g_{2}\right) \geq \operatorname{ord}\left(f_{0}\right)-1$. Actually, we are going to show that the condition imposed on the tangent cones implies that $\operatorname{ord}\left(g_{2}\right) \neq \operatorname{ord}\left(f_{0}\right)-1$, so that both ord $\left(g_{1}\right)$ and ord $\left(g_{2}\right)$ are greater than or equal to ord $\left(f_{0}\right)$ (which implies equimultiplicity).

So, suppose that ord $\left(g_{2}\right)=\operatorname{ord}\left(f_{0}\right)-1$. As ord $\left(g_{1}\right) \geq \operatorname{ord}\left(f_{0}\right)$, this implies that $\operatorname{ord}\left(g_{2}\right)<\operatorname{ord}\left(g_{1}\right)$. By Lemma 4.3 , there is an index $i_{0}$ such that the restriction of $\partial f / \partial z_{i_{0}}$ to $\operatorname{\Sigma in}\left(f_{0}\right) \times \mathbb{C}$ is not equal to 0 . Note also that, 
according to the hypothesis, the restriction of $\operatorname{in}\left(g_{2}\right)$ to $\Sigma \operatorname{in}\left(f_{0}\right)$ is also not equal to 0 . Pick a point $\left(\mathbf{z}_{0}, t_{0}\right) \in\left(\Sigma \operatorname{in}\left(f_{0}\right) \backslash\{\mathbf{0}\}\right) \times(D \backslash\{0\})$ such that, for all $s \neq 0$ sufficiently small,

$$
\operatorname{in}\left(\frac{\partial f}{\partial t}\right)\left(s \mathbf{z}_{0}, s t_{0}\right) \neq 0, \quad \operatorname{in}\left(g_{2}\right)\left(s \mathbf{z}_{0}\right) \neq 0, \quad \frac{\partial f}{\partial z_{i_{0}}}\left(s \mathbf{z}_{0}, s t_{0}\right) \neq 0
$$

and consider the curve $\gamma:(\mathbb{C}, 0) \rightarrow(B \times D,(\mathbf{0}, 0))$ defined by $s \mapsto \gamma(s)=$ $\left(\gamma_{1}(s), \gamma_{2}(s)\right)=\left(s \mathbf{z}_{0}, s t_{0}\right)$. Clearly, as $\left(\partial f / \partial z_{i_{0}}\right) \circ \gamma \neq 0$, the set $\gamma(\mathbb{C})$ is not contained in $\Sigma f$. Moreover,

$$
\operatorname{ord}\left(\frac{\partial f}{\partial t} \circ \gamma\right)=\operatorname{ord}\left(\frac{\partial f}{\partial t}\right)=\operatorname{ord}\left(g_{2}\right)+1=\operatorname{ord}\left(f_{0}\right)
$$

while

$$
\begin{aligned}
& \inf _{1 \leq i \leq n} \operatorname{ord}\left(\frac{\partial f}{\partial z_{i}} \circ \gamma\right) \\
& \quad \geq \inf _{1 \leq i \leq n}\left\{\operatorname{ord}\left(\frac{\partial f_{0}}{\partial z_{i}} \circ \gamma_{1}\right), \operatorname{ord}\left(\gamma_{2} \cdot \frac{\partial g_{1}}{\partial z_{i}} \circ \gamma_{1}\right), \operatorname{ord}\left(\gamma_{2}^{2} \cdot \frac{\partial g_{2}}{\partial z_{i}} \circ \gamma_{1}\right)\right\} \\
& \quad \geq \operatorname{ord}\left(f_{0}\right)
\end{aligned}
$$

(The second inequality comes from the relations $\gamma_{1}(\mathbb{C}) \subseteq \Sigma \operatorname{in}\left(f_{0}\right)$, ord $\left(g_{1}\right) \geq$ $\operatorname{ord}\left(f_{0}\right)$ and $\operatorname{ord}\left(g_{2}\right)=\operatorname{ord}\left(f_{0}\right)-1$.) In other words, the Thom inequality (see (2.1)) is not satisfied along the curve $\gamma$, and one gets a contradiction with Corollary 2.4.

REMARK 4.4. In fact, this proof of Theorem 4.2 shows that, for a family of the given form (i.e., $\left.f_{t}(\mathbf{z})=f_{0}(\mathbf{z})+t g_{1}(\mathbf{z})+t^{2} g_{2}(\mathbf{z})\right)$, if $f$ satisfies the Thom inequalities (see Definition 2.3) and if $\Sigma$ in $\left(f_{0}\right) \nsubseteq C\left(V\left(g_{2}\right)\right.$ ), then the family $\left\{f_{t}\right\}$ is equimultiple.

Second proof of Theorem 4.2. As in the second proof of Theorem 3.2, by [13, Corollary 4.6], if $0 \ll N_{1} \ll N_{2} \ll \cdots \ll N_{d}$ (where $\left.d=\operatorname{dim}_{0} \Sigma f_{t}\right)$, then the functions

$$
f_{t}+z_{1}^{N_{1}}+\cdots+z_{d}^{N_{d}}=f_{0}+z_{1}^{N_{1}}+\cdots+z_{d}^{N_{d}}+t g_{1}+t^{2} g_{2}
$$

form a $\mu$-constant family of isolated singularities as $t$ varies. As the integers $N_{1}, \ldots, N_{d}$ are arbitrarily large, $\operatorname{in}\left(f_{0}+z_{1}^{N_{1}}+\cdots+z_{d}^{N_{d}}\right)=\operatorname{in}\left(f_{0}\right)$, and therefore $\Sigma \operatorname{in}\left(f_{0}+z_{1}^{N_{1}}+\cdots+z_{d}^{N_{d}}\right) \nsubseteq C\left(V\left(g_{2}\right)\right)$. Hence, by Theorem 4.1, the family $\left\{f_{t}+z_{1}^{N_{1}}+\cdots+z_{d}^{N_{d}}\right\}$ is equimultiple. Again, as $N_{1}, \ldots, N_{d}$ are arbitrarily large, this implies that the family $\left\{f_{t}\right\}$ is also equimultiple. 
REMARK 4.5. Unlike the first proof, in this second approach one really needs to assume that the family $\left\{f_{t}\right\}$ is $\lambda_{\mathbf{z}}$-constant in order to apply [13, Corollary 4.6].

In the special case of a family with aligned singularities, Theorems 4.1 and 4.2 have the following corollary.

Corollary 4.6. Assume again that $f_{t}(\mathbf{z})=f_{0}(\mathbf{z})+t g_{1}(\mathbf{z})+t^{2} g_{2}(\mathbf{z})$, with $g_{2} \neq 0$. Also suppose here that, for all sufficiently small $t$, the function $f_{t}$ has a d-dimensional aligned singularity at $\mathbf{0}$. Under these conditions, if the family $\left\{f_{t}\right\}$ is topologically $V$-constant and such that $\Sigma \operatorname{in}\left(f_{0}\right) \nsubseteq C\left(V\left(g_{2}\right)\right)$, then it is equimultiple.

Proof. We proceed as in the proof of Corollary 3.6. If there exists a set of coordinates which is aligning for $f_{t}$ at $\mathbf{0}$, for all sufficiently small $t$, then, by $[13$, Corollary 7.8$]$ the family $\left\{f_{t}\right\}$ is $\lambda$-constant with respect to this coordinates system, and the result follows immediately from Theorem 4.2. Otherwise, as in the proof of [13, Theorem 7.9], we can show that, for $0 \ll N_{1} \ll N_{2} \ll \cdots \ll N_{d}$, the family of functions

$$
f_{t}+z_{1}^{N_{1}}+\cdots+z_{d}^{N_{d}}=f_{0}+z_{1}^{N_{1}}+\cdots+z_{d}^{N_{d}}+t g_{1}+t^{2} g_{2}
$$

is a $\mu$-constant family of isolated singularities. Then, we conclude as in the second proof of Theorem 4.2.

\section{$\S 5 . \quad \lambda_{\mathbf{z}}$-constant deformations and hyperplane sections}

In this section, we investigate the behavior of $\lambda_{\mathbf{z}}$-constant families under hyperplane sections. By Corollary 2.4, we know that $\lambda_{\mathbf{z}}$-constant families satisfy the Thom inequalities (see Definition 2.3). We show here that, under some appropriate conditions, hyperplane sections of $\lambda_{\mathbf{z}}$-constant families satisfy Thom's inequalities as well. Using this fact, we prove equimultiplicity for new families of nonisolated hypersurface singularities of the form $f_{t}(\mathbf{z})=$ $h_{t}\left(\mathbf{z}^{\prime}\right)+z_{n}^{2} k_{t}(\mathbf{z})$, where $\mathbf{z}^{\prime}:=\left(z_{1}, \ldots, z_{n-1}\right)$.

\subsection{Thom's inequalities and hyperplane sections}

Let $H$ be the hyperplane in $\mathbb{C}^{n}$ defined by $z_{i_{0}}=0$ for some $i_{0}$. Set

$$
\Xi f:=\left\{(\mathbf{z}, t) \in \mathbb{C}^{n} \times \mathbb{C} ; \frac{\partial f}{\partial z_{i}}(\mathbf{z}, t)=0,1 \leq i \leq n\right\}
$$

and

$$
\Xi\left(f_{\mid H \times \mathbb{C}}\right):=\left\{(\mathbf{z}, t) \in \mathbb{C}^{n} \times \mathbb{C} ; z_{i_{0}}=0, \frac{\partial f}{\partial z_{i}}(\mathbf{z}, t)=0,1 \leq i \leq n, i \neq i_{0}\right\} .
$$


We will denote by $\mathscr{I}(\Xi f \cap(H \times \mathbb{C}))$ the ideal in $\mathbb{C}\{\mathbf{z}, t\}$ generated by $\left\{z_{i_{0}}, \partial f / \partial z_{i} ; 1 \leq i \leq n\right\}$. Similarly, $\mathscr{I}\left(\Xi\left(f_{\mid H \times \mathbb{C}}\right)\right)$ will be the ideal in $\mathbb{C}\{\mathbf{z}, t\}$ generated by $\left\{z_{i_{0}}, \partial f / \partial z_{i} ; 1 \leq i \leq n, i \neq i_{0}\right\}$.

TheOREM 5.1. We suppose that $\mathscr{I}\left(\Xi\left(f_{\mid H \times \mathbb{C}}\right)\right)=\mathscr{I}(\Xi f \cap(H \times \mathbb{C}))$. In this case, if the family $\left\{f_{t}\right\}$ is $\lambda_{\mathbf{z}}$-constant, then, for any holomorphic curve

$$
\gamma:(\mathbb{C}, 0) \rightarrow((B \cap H) \times D,(\mathbf{0}, 0))
$$

not contained in $\Sigma\left(f_{\mid H \times \mathbb{C}}\right)$, we have

$$
\operatorname{ord}\left(\frac{\partial f_{\mid H \times \mathbb{C}}}{\partial t} \circ \gamma\right)>\inf _{\substack{1 \leq i \leq n \\ i \neq i_{0}}} \operatorname{ord}\left(\frac{\partial f_{\mid H \times \mathbb{C}}}{\partial z_{i}} \circ \gamma\right)
$$

In the special case of a family with isolated singularities, the result was first proved by Greuel and Pfister in [7, Lemma 3.1]. Note that, in this case, the conclusion (5.1) is equivalent to the $\mu$-constancy of the family $\left\{f_{t \mid H}\right\}$.

Proof of Theorem 5.1. It is similar to the proof of [7, Lemma 3.1]. Let

$$
\gamma:(\mathbb{C}, 0) \rightarrow((B \cap H) \times D,(\mathbf{0}, 0)), \quad s \mapsto \gamma(s)=\left(\gamma_{1}(s), \gamma_{2}(s)\right)
$$

be any holomorphic curve not contained in $\Sigma\left(f_{\mid H \times \mathbb{C}}\right)$. As $\mathscr{I}\left(\Xi\left(f_{\mid H \times \mathbb{C}}\right)\right)=$ $\mathscr{I}(\Xi f \cap(H \times \mathbb{C}))$, the partial derivative $\partial f / \partial z_{i_{0}}$ must belong to $\mathscr{I}\left(\Xi\left(f_{\mid H \times \mathbb{C}}\right)\right)$, and since $\gamma(\mathbb{C}) \subseteq H \times D$, this implies that

$$
\operatorname{ord}\left(\frac{\partial f}{\partial z_{i_{0}}} \circ \gamma\right) \geq \inf _{\substack{1 \leq i \leq n \\ i \neq i_{0}}} \operatorname{ord}\left(\frac{\partial f}{\partial z_{i}} \circ \gamma\right)
$$

Now, as $\left\{f_{t}\right\}$ is $\lambda_{\mathbf{z}}$-constant, Corollary 2.4 shows that

$$
\operatorname{ord}\left(\frac{\partial f}{\partial t} \circ \gamma\right)>\inf _{1 \leq i \leq n} \operatorname{ord}\left(\frac{\partial f}{\partial z_{i}} \circ \gamma\right) \stackrel{(5.2)}{=} \inf _{\substack{1 \leq i \leq n \\ i \neq i_{0}}} \operatorname{ord}\left(\frac{\partial f}{\partial z_{i}} \circ \gamma\right)
$$

and since $\gamma(s) \in H \times D$, we have (5.1).

REMARK 5.2. In fact, the proof of Theorem 5.1 shows that, under the hypothesis $\mathscr{I}\left(\Xi\left(f_{\mid H \times \mathbb{C}}\right)\right)=\mathscr{I}(\Xi f \cap(H \times \mathbb{C}))$, if $f$ satisfies the Thom inequalities (2.1), then $f_{\mid H \times \mathbb{C}}$ satisfies the Thom inequalities (5.1). 
5.2. Deformations of the form $f_{t}(\mathbf{z})=h_{t}\left(\mathbf{z}^{\prime}\right)+z_{n}^{2} k_{t}(\mathbf{z})$

Throughout this section, we assume that $f$ can be written in the form $f(\mathbf{z}, t)=h\left(\mathbf{z}^{\prime}, t\right)+z_{n}^{2} k(\mathbf{z}, t)$, where $h$ and $k$ are holomorphic functions and $\mathbf{z}^{\prime}:=\left(z_{1}, \ldots, z_{n-1}\right)$. As usual, we set $h_{t}\left(\mathbf{z}^{\prime}\right):=h\left(\mathbf{z}^{\prime}, t\right)$ and $k_{t}(\mathbf{z}):=k(\mathbf{z}, t)$. We will assume that $h_{t}$ is reduced and that $n \geq 3$. The interest in families of this form (already studied by Greuel and Pfister in [7] in the special case of isolated singularities) is that they satisfy the condition $\mathscr{I}\left(\Xi\left(f_{\mid H \times \mathbb{C}}\right)\right)=$ $\mathscr{I}(\Xi f \cap(H \times \mathbb{C}))$ of Theorem 5.1 , with the hyperplane $H$ defined by $z_{n}=0$. Then, by this theorem or by [7, Lemma 3.1], in many interesting cases the equimultiplicity problem for the family $\left\{f_{t}\right\}$ can be reduced to one for the family $\left\{h_{t}\right\}$.

For example, let us consider the following four conditions:

(C1) for all $t$ sufficiently small, $h_{t}$ is convenient and has a nondegenerate Newton principal part with respect to $\mathbf{z}^{\prime}$ (see [9] for the definition);

(C2) for all $t$ sufficiently small, $h_{t}$ is of the form

$$
h_{t}\left(\mathbf{z}^{\prime}\right)=h_{0}\left(\mathbf{z}^{\prime}\right)+\xi(t) \ell\left(\mathbf{z}^{\prime}\right),
$$

where $\xi$ and $\ell$ are holomorphic functions with $\xi(0)=0$;

(C3) $h_{0}$ is a semiquasihomogeneous polynomial with respect to $\mathbf{z}^{\prime}$;

(C4) $n=3$ (i.e., $\left\{h_{t}\right\}$ is a family of plane curve singularities).

In [3], the first author proved the following equimultiplicity result for nonisolated singularities.

TheOREM 5.3 ([3, Theorem 2]). Suppose that $\left\{f_{t}\right\}$ is a topologically $V$-constant family of d-dimensional aligned singularities, at $\mathbf{0}$, of the form $f_{t}(\mathbf{z})=h_{t}\left(\mathbf{z}^{\prime}\right)+z_{n}^{2} k_{t}(\mathbf{z})$. Let $\left\{t_{k}\right\}$ be an infinite sequence of points in $\mathbb{C}$ approaching the origin. Suppose that the coordinates $\mathbf{z}=\left(z_{1}, \ldots, z_{n}\right)$, or some cyclic permutation of them, form an aligning set of coordinates at $\mathbf{0}$ for $f_{0}$ and for $f_{t_{k}}$ for all $k$. Under these conditions, if at least one of the four conditions (C1)-(C4) above is satisfied, then the family $\left\{h_{t}\right\}$ is equimultiple. In particular, if, in addition, $\operatorname{ord}\left(h_{t}\right) \leq \operatorname{ord}\left(z_{n}^{2} k_{t}\right)$, then the family $\left\{f_{t}\right\}$ is equimultiple too.

In the special case of a family with isolated singularities, the result was first proved by Greuel and Pfister in [7, Proposition 3.2].

Actually, the proof of Theorem 5.3 shows the next result.

THEOREM 5.4. Suppose that the family $\left\{f_{t}\right\}$ is $\lambda_{\mathbf{z}}$-constant and of the form $f_{t}(\mathbf{z})=h_{t}\left(\mathbf{z}^{\prime}\right)+z_{n}^{2} k_{t}(\mathbf{z})$. If at least one of the four conditions (C1)(C4) above is satisfied, then the family $\left\{h_{t}\right\}$ is equimultiple. 
As above, if, in addition to the hypotheses of Theorem 5.4, we also have $\operatorname{ord}\left(h_{t}\right) \leq \operatorname{ord}\left(z_{n}^{2} k_{t}\right)$, then the family $\left\{f_{t}\right\}$ is equimultiple too. (A similar remark applies to the theorems below as well.)

Proof of Theorem 5.4. By [13, Corollary 4.6], for all integers $0 \ll N_{1} \ll$ $N_{2} \ll \cdots \ll N_{d}$ (where $d=\operatorname{dim}_{0} \Sigma f_{t}$ ), the family $\left\{f_{t}+z_{1}^{N_{1}}+\cdots+z_{d}^{N_{d}}\right\}$ is a $\mu$-constant family of isolated singularities. Applying [7, Lemma 3.1], with the hyperplane $z_{n}=0$, gives that the family $\left\{h_{t}+z_{1}^{N_{1}}+\cdots+z_{d}^{N_{d}}\right\}$ is also a $\mu-$ constant family of isolated singularities. As $N_{1}, \ldots, N_{d}$ are arbitrarily large, if $h_{t}$ satisfies one of the conditions (C1)-(C4), then so does $h_{t}+z_{1}^{N_{1}}+\cdots+$ $z_{d}^{N_{d}}$. But in each case, $(\mathrm{C} 1)-(\mathrm{C} 4)$, the Zariski multiplicity conjecture is true if we deal with families of isolated singularities (the references for that are [1], [6], [15], [17], [19], [21]). In other words, the family $\left\{h_{t}+z_{1}^{N_{1}}+\cdots+z_{d}^{N_{d}}\right\}$ is equimultiple. Again, as $N_{1}, \ldots, N_{d}$ are arbitrarily large, we deduce that the family $\left\{h_{t}\right\}$ is also equimultiple.

In the same vein, we can also prove the next theorem.

TheOREM 5.5. Suppose that the family $\left\{f_{t}\right\}$ is $\lambda_{\mathbf{z}}$-constant and of the form $f_{t}(\mathbf{z})=h_{t}\left(\mathbf{z}^{\prime}\right)+z_{n}^{2} k_{t}(\mathbf{z})$, where $h_{t}\left(\mathbf{z}^{\prime}\right)=h_{0}\left(\mathbf{z}^{\prime}\right)+t \ell_{1}\left(\mathbf{z}^{\prime}\right)+t^{2} \ell_{2}\left(\mathbf{z}^{\prime}\right)$ for some holomorphic functions $\ell_{1}$ and $\ell_{2}$, with $\ell_{2} \neq 0$. Suppose also that $\Sigma \operatorname{in}\left(h_{0}\right) \nsubseteq C\left(V\left(\ell_{2}\right)\right)$. Under these conditions, the family $\left\{h_{t}\right\}$ is equimultiple.

To prove this theorem, we can proceed exactly as in the proof of Theorem 5.4, first by applying the Massey-Iomdine-Lê formula and the GreuelPfister lemma in order to reduce the problem to one dealing with $\mu$-constant families of isolated singularities, and then by invoking Theorem 4.1. The details of this proof are left to the reader. Theorem 5.5 can be also obtained as a corollary of Theorem 5.1 (or Remark 5.2) and Remark 4.4. With this second approach, the $\lambda_{\mathbf{z}}$-constancy of $\left\{f_{t}\right\}$, in Theorem 5.5, can be replaced by the condition " $f$ satisfies the Thom inequalities" (see Definition 2.3). The details are as follows.

Proof of Theorem 5.5. By Theorem 5.1 (or Remark 5.2), applied to the family $\left\{f_{t}\right\}$ and the hyperplane $H$ defined by $z_{n}=0$, for any holomorphic curve $\gamma:(\mathbb{C}, 0) \rightarrow((B \cap H) \times D,(\mathbf{0}, 0))$ not contained in $\Sigma\left(f_{\mid H \times \mathbb{C}}\right) \approx \Sigma h$, the 
Thom inequality (5.1) holds. That is, by abuse of notation, ${ }^{\dagger}$

$$
\operatorname{ord}\left(\frac{\partial h}{\partial t} \circ \gamma\right)>\inf _{1 \leq i \leq n-1} \operatorname{ord}\left(\frac{\partial h}{\partial z_{i}} \circ \gamma\right) .
$$

As $\operatorname{\Sigma in}\left(h_{0}\right) \nsubseteq C\left(V\left(\ell_{2}\right)\right)$, this implies that the family $\left\{h_{t}\right\}$ is equimultiple (see Remark 4.4).

Focusing on aligned singularities, we get the following result.

THEOREM 5.6. Suppose that $\left\{f_{t}\right\}$ is a topologically $V$-constant family of d-dimensional aligned singularities, at $\mathbf{0}$, of the form $f_{t}(\mathbf{z})=h_{t}\left(\mathbf{z}^{\prime}\right)+$ $z_{n}^{2} k_{t}(\mathbf{z})$, where $h_{t}\left(\mathbf{z}^{\prime}\right)=h_{0}\left(\mathbf{z}^{\prime}\right)+t \ell_{1}\left(\mathbf{z}^{\prime}\right)+t^{2} \ell_{2}\left(\mathbf{z}^{\prime}\right)$ for some holomorphic functions $\ell_{1}$ and $\ell_{2}$, with $\ell_{2} \neq 0$. Let $\left\{t_{k}\right\}$ be an infinite sequence of points in $\mathbb{C}$ approaching the origin. Suppose that the coordinates $\mathbf{z}=\left(z_{1}, \ldots, z_{n}\right)$, or some cyclic permutation of them, form an aligning set of coordinates at 0 for $f_{0}$ and for $f_{t_{k}}$ for all $k$. Finally, suppose that $\Sigma \operatorname{in}\left(h_{0}\right) \nsubseteq C\left(V\left(\ell_{2}\right)\right)$. Under these conditions, the family $\left\{h_{t}\right\}$ is equimultiple.

Proof. As cyclic permutations of the coordinates do not alter the special form of the function $f_{t}$, we can suppose that the aligning set of coordinates for $f_{0}$ and for $f_{t_{k}}$, for all $k$, is the set $\mathbf{z}=\left(z_{1}, \ldots, z_{n}\right)$. Then, by [13, Corollary 7.8], $\lambda_{f_{t_{k}, \mathbf{z}}}^{i}(\mathbf{0})=\lambda_{f_{0}, \mathbf{z}}^{i}(\mathbf{0})$ for all large $k$. As in the proof of Corollary 3.6, this implies that, for all integers $0 \ll N_{1} \ll N_{2} \ll \cdots \ll N_{d}$, the functions $f_{t}+z_{1}^{N_{1}}+\cdots+z_{d}^{N_{d}}$ form a $\mu$-constant family of isolated singularities as $t$ varies. Then, applying [7, Lemma 3.1], with the hyperplane $z_{n}=0$, gives that the family $\left\{h_{t}+z_{1}^{N_{1}}+\cdots+z_{d}^{N_{d}}\right\}$ is also a $\mu$-constant family of isolated singularities. As $N_{1}, \ldots, N_{d}$ are arbitrarily large, $\operatorname{in}\left(h_{0}+z_{1}^{N_{1}}+\cdots+z_{d}^{N_{d}}\right)=\operatorname{in}\left(h_{0}\right)$, and therefore $\Sigma \operatorname{in}\left(h_{0}+z_{1}^{N_{1}}+\cdots+z_{d}^{N_{d}}\right) \nsubseteq C\left(V\left(\ell_{2}\right)\right)$. Hence, by Theorem 4.1, the family $\left\{h_{t}+z_{1}^{N_{1}}+\cdots+z_{d}^{N_{d}}\right\}$ is equimultiple. Again, as $N_{1}, \ldots, N_{d}$ are arbitrarily large, we deduce that the family $\left\{h_{t}\right\}$ is also equimultiple.

\section{Appendix A. Lê numbers}

The Lê numbers generalize to nonisolated hypersurface singularities the data given by the Milnor number for an isolated singularity. They were introduced about 25 years ago by Massey. (For a detailed exposition, see [13], [14]). For its convenience, we also briefly recall the definition hereafter.

\footnotetext{
${ }^{\dagger}$ We have identified the $(n+1)$-tuple $\gamma(s)=\left(\gamma_{1,1}(s), \ldots, \gamma_{1, n-1}(s), 0, \gamma_{2}(s)\right)$ with the $n$-tuple $\left(\gamma_{1,1}(s), \ldots, \gamma_{1, n-1}(s), \gamma_{2}(s)\right)$.
} 
The Lê numbers are intersection numbers of certain analytic cycles (socalled Lê cycles) with certain affine subspaces. The definition of the Lê cycles, in turn, is based on the notion of gap sheaf. We start this section by explaining what analytic cycle and gap sheaf mean.

\section{A.1 Analytic cycles}

Let $\left(X, \mathscr{O}_{X}\right)$ be a complex analytic space. An analytic cycle in $X$ is a finite formal sum $\sum m_{V}[V]$, where the $V$ 's are distinct irreducible analytic subsets of $X$ and where the $m_{V}$ 's are nonzero integers. The analytic cycle associated to $X$ is the cycle $[X]:=\sum m_{V}[V]$ obtained when the $V$ 's run over all the irreducible components of $X$ and when the $m_{V}$ 's are defined as follows. Take a point $\mathbf{x} \in V$, and consider the germ $V_{\mathbf{x}}$ of $V$ at $\mathbf{x}$. Then, if $V_{\mathbf{x}}^{0}$ is any irreducible germ component of $V_{\mathbf{x}}$, the integer $m_{V}$ is defined to be the length of the local ring of $X$ at $\mathbf{x}$ localized at the prime ideal corresponding to $V_{\mathbf{x}}^{0}$. Of course, this definition is independent of the choices of the point $\mathbf{x}$ and the component $V_{\mathbf{x}}^{0}$. (Hereafter, we will always use brackets [.] to denote analytic cycles.)

If $V$ and $W$ are irreducible analytic subsets of a connected complex analytic manifold $M$, then we say that $V$ and $W$ intersect properly in $M$ if, for each irreducible component $Z$ of $V \cap W$, we have

$$
\operatorname{codim}_{M} Z=\operatorname{codim}_{M} V+\operatorname{codim}_{M} W
$$

When this is the case, the intersection product of $[V]$ and $[W]$ in $M$ (denoted by $[V] \cdot[W])$ is characterized axiomatically by four properties: openness, transversality, projection, and continuity (see [14, Appendix A] and [5, Example 11.4.4] for details). Now, if $\sum m_{i}\left[V_{i}\right]$ and $\sum n_{j}\left[W_{j}\right]$ are two cycles in $M$ intersecting properly (i.e., $V_{i}$ and $W_{j}$ intersect properly for all $i, j$ ), then we define their intersection product as

$$
\left(\sum m_{i}\left[V_{i}\right]\right) \cdot\left(\sum n_{j}\left[W_{j}\right]\right):=\sum m_{i} n_{j}\left(\left[V_{i}\right] \cdot\left[W_{j}\right]\right)
$$

Finally, if $C_{1}$ and $C_{2}$ are two cycles intersecting properly and if

$$
C_{1} \cdot C_{2}=\sum p_{k}\left[Z_{k}\right]
$$

then the intersection number $\left(C_{1} \cdot C_{2}\right)_{Z_{k}}$ of $C_{1}$ and $C_{2}$ at $Z_{k}$ is defined by

$$
\left(C_{1} \cdot C_{2}\right)_{Z_{k}}:=p_{k}
$$

In other words, $\left(C_{1} \cdot C_{2}\right)_{Z_{k}}$ represents the number of times $Z_{k}$ occurs in the intersection, counted with multiplicity. 


\section{A.2 Gap sheaves}

Let $\left(X, \mathscr{O}_{X}\right)$ be a complex analytic space, let $W \subseteq X$ be an analytic subset of $X$, and let $\mathscr{I}$ be a coherent sheaf of ideals in $\mathscr{O}_{X}$. As usual, we will denote by $V(\mathscr{I})$ the analytic space defined by the vanishing of $\mathscr{I}$. At each point $\mathbf{x} \in V(\mathscr{I})$ we want to consider scheme-theoretically those components of $V(\mathscr{I})$ which are not contained in $W$. For this purpose, we look at a minimal primary decomposition of the stalk $\mathscr{I}_{\mathbf{x}}$ of $\mathscr{I}$ in the local ring $\mathscr{O}_{X, \mathbf{x}}$, and we consider the ideal $\mathscr{I}_{\mathbf{x}} \neg W$ in $\mathscr{O}_{X, \mathbf{x}}$ consisting of the intersection of those (possibly embedded) primary components $Q$ of $\mathscr{I}_{\mathbf{x}}$ such that $V(Q) \nsubseteq W$. Note that if $S$ is the multiplicatively closed set

$$
\mathscr{O}_{X, \mathbf{x}} \backslash \bigcup P
$$

where the union is taken over all the associated prime ideals $P$ of $\mathscr{O}_{X, \mathbf{x}} / \mathscr{I}_{\mathbf{x}}$ such that $V(P) \nsubseteq W$, then

$$
\mathscr{I}_{\mathbf{x}} \neg W=S^{-1} \mathscr{I}_{\mathbf{x}} \cap \mathscr{O}_{X, \mathbf{x}}
$$

where $S^{-1} \mathscr{I}_{\mathbf{x}}$ is the ring of fractions of $\mathscr{I}_{\mathbf{x}}$ with denominators in $S$. In particular, the definition of $\mathscr{I}_{\mathbf{x}} \neg W$ does not depend on the choice of the minimal primary decomposition of $\mathscr{I}_{\mathbf{x}}$. Now, if we perform the operation described above at the point $\mathbf{x}$ simultaneously at all points of $V(\mathscr{I})$, then we obtain a coherent sheaf of ideals called a gap sheaf and denoted by $\mathscr{I} \neg W$. Hereafter, we shall denote by $V(\mathscr{I}) \neg W$ the scheme (i.e., the complex analytic space) $V(\mathscr{I} \neg W)$ defined by the vanishing of the gap sheaf $\mathscr{I} \neg W$.

\section{A.3 Lê cycles and Lê numbers}

Now, we are ready to define the Lê cycles and the Lê numbers. Consider a holomorphic function $h:(U, \mathbf{0}) \rightarrow(\mathbb{C}, 0)$, where $U$ is an open neighborhood of $\mathbf{0}$ in $\mathbb{C}^{n}$, and fix a system of linear coordinates $\mathbf{z}=\left(z_{1}, \ldots, z_{n}\right)$ for $\mathbb{C}^{n}$. Let $\Sigma h$ be the critical locus of $h$. For $0 \leq i \leq n-1$, the ith (relative) polar variety of $h$ with respect to $\mathbf{z}$ is the scheme

$$
\Gamma_{h, \mathbf{z}}^{i}:=V\left(\frac{\partial h}{\partial z_{i+1}} \cdots \frac{\partial h}{\partial z_{n}}\right) \neg^{\Sigma h} .
$$

Clearly, for $i=0, \Gamma_{h, \mathbf{z}}^{0}=\emptyset$. Also, it can be shown easily that

$$
\left(\Gamma_{h, \mathbf{z}}^{i+1} \cap V\left(\frac{\partial h}{\partial z_{i+1}}\right)\right) \neg \Sigma h=\Gamma_{h, \mathbf{z}}^{i}
$$


as schemes, and therefore, all the components of the cycle

$$
\left[\Gamma_{h, \mathbf{z}}^{i+1} \cap V\left(\frac{\partial h}{\partial z_{i+1}}\right)\right]-\left[\Gamma_{h, \mathbf{z}}^{i}\right]
$$

are contained in $\Sigma h$. The cycle (A.1) is called the ith Lê cycle of $h$ with respect to $\mathbf{z}$. It is denoted by $\left[\Lambda_{h, \mathbf{z}}^{i}\right]$.

Definition A.1. The ith Lê number $\lambda_{h, \mathbf{z}}^{i}(\mathbf{p})$ of $h$ at $\mathbf{p}=\left(p_{1}, \ldots, p_{n}\right)$ with respect to the coordinates system $\mathbf{z}$ is defined to be the intersection number

$$
\lambda_{h, \mathbf{z}}^{i}(\mathbf{p}):=\left(\left[\Lambda_{h, \mathbf{z}}^{i}\right] \cdot\left[V\left(z_{1}-p_{1}, \ldots, z_{i}-p_{i}\right)\right]\right)_{\mathbf{p}}
$$

provided that this intersection is 0-dimensional or empty at $\mathbf{p}$; otherwise, we say that $\lambda_{h, \mathbf{z}}^{i}(\mathbf{p})$ is undefined.

For $i=0$, the relation (A.2) means that

$$
\lambda_{h, \mathbf{z}}^{0}(\mathbf{p})=\left(\left[\Lambda_{h, \mathbf{z}}^{0}\right] \cdot U\right)_{\mathbf{p}}=\left[\Gamma_{h, \mathbf{z}}^{1} \cap V\left(\frac{\partial h}{\partial z_{1}}\right)\right]_{\mathbf{p}} .
$$

The last term is also equal to the intersection number

$$
\left(\left[\Gamma_{h, \mathbf{z}}^{1}\right] \cdot\left[V\left(\frac{\partial h}{\partial z_{1}}\right)\right]\right)_{\mathbf{p}}
$$

whenever $\Gamma_{h, \mathbf{z}}^{1}$ is 1 -dimensional at $\mathbf{p}$.

Remark A.2. The Lê numbers have the following properties.

(1) For a generic choice of coordinates all the Lê numbers are defined.

(2) For $\operatorname{dim}_{\mathbf{p}} \Sigma h<i \leq n-1, \lambda_{h, \mathbf{z}}^{i}(\mathbf{p})=0$. For this reason, we usually only consider the Lê numbers

$$
\lambda_{h, \mathbf{z}}^{0}(\mathbf{p}), \ldots, \lambda_{h, \mathbf{z}}^{\operatorname{dim}_{\mathbf{p}} \Sigma h}(\mathbf{p}) .
$$

(3) Finally, if $\mathbf{p}$ is an isolated singularity of $h$, then $\lambda_{h, \mathbf{z}}^{0}(\mathbf{p})$ (which is the only possible nonzero Lê number) equals the Milnor number of $h$ at $\mathbf{p}$.

\section{Appendix B. Aligned singularities}

Aligned singularities were also introduced by Massey in [13]. They include isolated singularities and line singularities. To give the definition, we first need to introduce the notion of good stratification. 
Let $h:(U, \mathbf{0}) \rightarrow(\mathbb{C}, 0)$ be a holomorphic function, where $U$ is an open neighborhood of $\mathbf{0}$ in $\mathbb{C}^{n}$. A good stratification for $h$ at $\mathbf{0}$ is an analytic stratification $\mathscr{S}$ of the hypersurface $V(h)$ in a neighborhood $W \subseteq U$ of $\mathbf{0}$ such that

(1) the smooth part of $V(h)$ is a stratum;

(2) $\mathscr{S}$ satisfies Thom's $a_{h}$ condition with respect to $W \backslash V(h)$; that is, if $\left\{\mathbf{p}_{k}\right\}$ is a sequence of points in $W \backslash V(h)$ such that $\mathbf{p}_{k} \rightarrow \mathbf{p} \in S$ (where $S$ is a stratum) and the tangent space $T_{\mathbf{p}_{k}} V\left(h-h\left(\mathbf{p}_{k}\right)\right) \rightarrow T$, then $T_{\mathbf{p}} S \subseteq T$.

Such a stratification always exists (see [8]).

An aligned good stratification for $h$ at $\mathbf{0}$ is a good stratification for $h$ at $\mathbf{0}$ in which the closure of each stratum of the singular set $\Sigma h$ of $h$ is smooth at the origin. If such an aligned good stratification exists and if $\operatorname{dim}_{\mathbf{0}} \Sigma h=d$, then we say that $h$ has a $d$-dimensional aligned singularity at $\mathbf{0}$.

Now, if $\mathscr{S}$ is an aligned good stratification for $h$ at $\mathbf{0}$, then we say that a linear choice of coordinates $\mathbf{z}=\left(z_{1}, \ldots, z_{n}\right)$ is an aligning set of coordinates for $\mathscr{S}$ if, for each $i$, the $(n-i)$-plane $V\left(z_{1}, \ldots, z_{i}\right)$ in $\mathbb{C}^{n}$ intersects transversely the closure of each stratum of dimension at least $i$ at the origin. We say that a set of coordinates $\mathbf{z}=\left(z_{1}, \ldots, z_{n}\right)$ is aligning for $h$ at $\mathbf{0}$ if there exists an aligned good stratification for $h$ at $\mathbf{0}$ with respect to which $\mathbf{z}$ is aligning.

REMARK B.1. Given an aligned singularity, aligning sets of coordinates are generic.

Our main interest in aligned singularities and aligning sets of coordinates comes from the following theorem by Massey.

Theorem B.2 ([13, Corollary 7.8]). Let $U$ be an open neighborhood of $\mathbf{0}$ in $\mathbb{C}^{n}$, and let $h_{1}, h_{2}:(U, \mathbf{0}) \rightarrow(\mathbb{C}, 0)$ be two reduced holomorphic functions with a d-dimensional aligned singularity at $\mathbf{0}$. Let $\mathbf{z}$ and $\tilde{\mathbf{z}}$ be aligning sets of coordinates at $\mathbf{0}$ for $h_{1}$ and $h_{2}$, respectively. If $h_{1}$ and $h_{2}$ are topologically $V$-equivalent (i.e., if there exist open neighborhoods $U_{1}, U_{2} \subseteq U$ around the origin together with a homeomorphism $\varphi:\left(U_{1}, \mathbf{0}\right) \rightarrow\left(U_{2}, \mathbf{0}\right)$ such that $\left.\varphi\left(V\left(h_{1}\right) \cap U_{1}\right)=V\left(h_{2}\right) \cap U_{2}\right)$, then

$$
\lambda_{h_{1}, \mathbf{z}}^{i}(\mathbf{0})=\lambda_{h_{2}, \tilde{\mathbf{z}}}^{i}(\mathbf{0})
$$

for all $i$. 
Acknowledgments. The authors thank the referees for valuable comments and suggestions that led to improvements in this paper. The authors' research was partially supported by the Conselho Nacional de Desenvolvimento Científico e Tecnológico (CNPq), Brazil.

\section{REFERENCES}

[1] O. M. Abderrahmane, On the deformation with constant Milnor number and Newton polyhedron, preprint, http://www.rimath.saitama-u.ac.jp/lab.jp/Fukui /ould/dahm.pdf (accessed 21 November 2004).

[2] J. Fernández de Bobadilla and T. Gaffney, The Lê numbers of the square of a function and their applications, J. Lond. Math. Soc. (2) 77 (2008), 545-557. MR 2418291. DOI $10.1112 / \mathrm{jlms} / \mathrm{jdm} 101$.

[3] C. Eyral, Zariski's multiplicity question and aligned singularities, C. R. Math. Acad. Sci. Paris 342 (2006), 183-186. MR 2198190. DOI 10.1016/j.crma.2005.12.008.

[4] - Zariski's multiplicity question - A survey, New Zealand J. Math. 36 (2007), 253-276. MR 2476643.

[5] W. Fulton, Intersection Theory, Ergeb. Math. Grenzgeb. (3) 2, Springer, Berlin, 1984. MR 0732620. DOI 10.1007/978-3-662-02421-8.

[6] G.-M. Greuel, Constant Milnor number implies constant multiplicity for quasihomogeneous singularities, Manuscripta Math. 56 (1986), 159-166. MR 0850367. DOI 10.1007/BF01172153.

[7] G.-M. Greuel and G. Pfister, Advances and improvements in the theory of standard bases and syzygies, Arch. Math. (Basel) 66 (1996), 163-176. MR 1367159. DOI $10.1007 / \mathrm{BF} 01273348$.

[8] H. A. Hamm and Lê Dũng Tráng, Un théorème de Zariski du type de Lefschetz, Ann. Sci. Éc. Norm. Supér. (4) 6 (1973), 317-355. MR 0401755.

[9] A. G. Kouchnirenko, Polyèdres de Newton et nombres de Milnor, Invent. Math. 32 (1976), 1-31. MR 0419433.

[10] Lê Dũng Tráng, "Topologie des singularités des hypersurfaces complexes" in Singularités à Cargèse (Cargèse, 1972), Astérisque 7/8, Soc. Math. France, Paris, 1973, 171-182. MR 0361147.

[11] Lê Dũng Tráng and C. P. Ramanujam, The invariance of Milnor number implies the invariance of the topological type, Amer. J. Math. 98 (1976), 67-78. MR 0399088.

[12] Lê Dũng Tráng and K. Saito, La constance du nombre de Milnor donne des bonnes stratifications, C. R. Acad. Sci. Paris Sér. A-B 277 (1973), 793-795. MR 0350063.

[13] D. B. Massey, Lê cycles and hypersurface singularities, Lecture Notes in Math. 1615, Springer, Berlin, 1995. MR 1441075.

[14] - Numerical Control over Complex Analytic Singularities, Mem. Amer. Math. Soc. 163 (2003), no. 778. MR 1962934. DOI 10.1090/memo/0778.

[15] D. O'Shea, Topologically trivial deformations of isolated quasihomogeneous hypersurface singularities are equimultiple, Proc. Amer. Math. Soc. 101 (1987), 260-262. MR 0902538. DOI 10.2307/2045992.

[16] C. Plénat and D. Trotman, On the multiplicities of families of complex hypersurfacegerms with constant Milnor number, Internat. J. Math. 24 (2013), article no. 1350021. MR 3048008. DOI 10.1142/S0129167X13500213. 
[17] M. J. Saia and J. N. Tomazella, Deformations with constant Milnor number and multiplicity of complex hypersurfaces, Glasg. Math. J. 46 (2004), 121-130. MR 2034839. DOI 10.1017/S0017089503001599.

[18] B. Teissier, "Cycles évanescents, sections planes et conditions de Whitney" in Singularités à Cargèse (Cargèse, 1972), Astérisque 7/8, Soc. Math. France, Paris, 1973, 285-362. MR 0374482.

[19] D. Trotman, Partial results on the topological invariance of the multiplicity of a complex hypersurface, lecture, Université Paris 7, France, 1977.

[20] O. Zariski, Some open questions in the theory of singularities, Bull. Amer. Math. Soc. 77 (1971), 481-491. MR 0277533.

[21] - On the topology of algebroid singularities, Amer. J. Math. 54 (1932), 453465. MR 1507926. DOI 10.2307/2370887.

Christophe Eyral

Institute of Mathematics

Polish Academy of Sciences

00-656 Warsaw

Poland

eyralchr@yahoo.com

Maria Aparecida Soares Ruas

Instituto de Ciências Matemáticas e de Computação

Universidade de São Paulo

13566-590 São Carlos - SP

Brazil

maasruas@icmc.usp.br 